\title{
Leucines on a roll
}



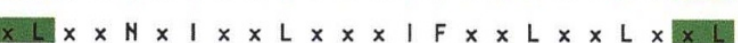

Bostjan Kobe

Nature Structural Biology, 3, no 12, 977-980 (1996).

Figure 3 was inadvertently misprinted. The version to the right is as it should have appeared in the December issue of Nature Stuctural Biology.

Fig. 3 Consensus sequences of repeats in proteins with coiled folds. Structural units in $\alpha / \alpha$ coiled folds and right-handed $\beta$-helices do not shown any identifiable sequence repeat, although attempts have been made to define a structural profile for the latter 33 . $L \beta H$, left-handed $\beta$-helix. The approximate locations of the elements of secondary structure are shown above the sequence: "a", $\alpha$-helix; " $b$ ", $\beta$-strand; " $3_{10}$ ", $3_{10}$-helix; "I", loop. Some consensus residues that perform similar roles in different coiled folds are coloured: yellow, hydrophobic residues that form the hydrophobic core of the coil; magenta, polar residues that form hydrogen bonds with neighbouring repeats, such as in asparagine ladders ${ }^{7}$; red and blue, Asp and Arg residues that form a ladder in LRV; cyan, residues that coordinate $\mathrm{Ca}^{2+}$ ion in the $\beta$-roll structures. Two consensus sequences are shown for LRR proteins corresponding to two subfamilies identified by sequence-profile searching 30: the longer $~ 28$-residue LRRs found in RI (LRR-28), and the "typical" shorter LRRs with $\sim 24$ residues found in $>90 \%$ of LRR proteins for which no structural information is currently available (LRR-24). The important differences between LRR and LRV consensus sequences, the XLXL motif verusus the LxxL motif, respectively, are highlighted with a green box. The 'phasing' of LRRs (that is, what residue corresponds to the beginning of a structural unit) has been depicted rather arbitrarily in the literature. Here I use a phasing different from that of Peters et al. ${ }^{2}$, not with the intention of confusing the readers, but to illustrate the unit most compatible with genetic and biochemical data such as the exon/intron boundaries and the neurotropin binding activities of single motifs from neurotropin receptors (refs 35,36; M.E. Lang, B.K., J.M. Windisch, R. Marksteiner, E. Schneider-Scherzer, M. Schweiger and R. Schneider, personal communication).

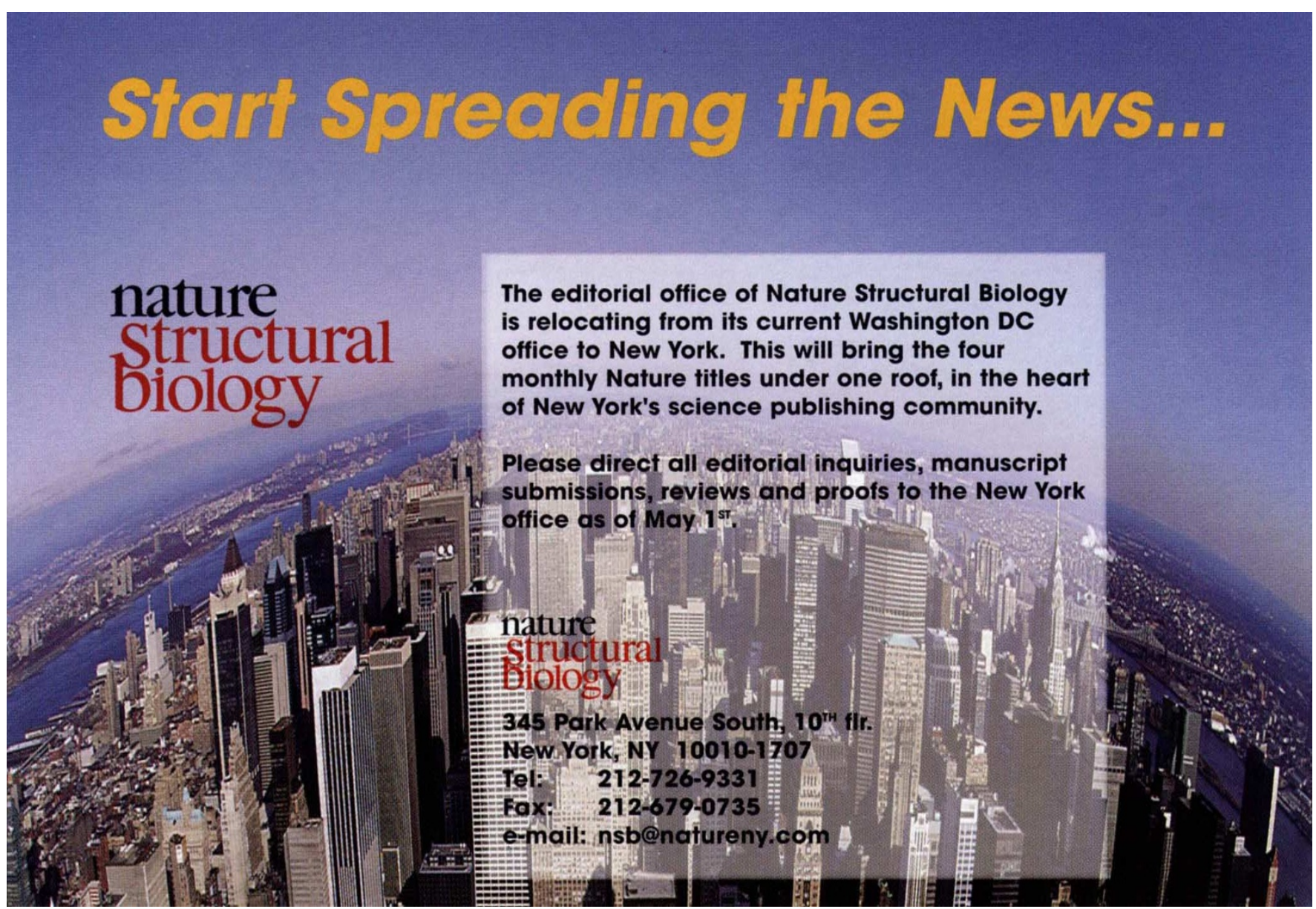

